has been heavily deposited. The Robinsons suggest that the silk may in fact prevent the female from bending at the waist, which would in turn prevent her from picking off the male while he is copulating.

As well as describing the unique courtship, the Robinsons also describe the kleptoparasites of Nephila, spiders of other species which live in the web and steal food caught by the host. Often, the kleptoparasites were seen to rush in and start feeding while Nephila was actually subduing or wrapping its prey, a somewhat hazardous past-time as the kleptoparasites themselves often became enmeshed.

\section{Origin of antibody diversity}

\section{from a Correspondent}

THE apparently inexhaustible repertoire of antibody-combining sites possessed by vertebrate animals raises an important question. Does the germ line genome code for all antibody genes (germ line theory), or is diversity generated during the life of an individual by mutation of a restricted number of germ line genes (somatic theory)? Argument surrounding this question has raged backwards and forwards ever since the discovery that heavy and light chains of immunoglobulin molecules are composed of constant (C) and variable (V) regions (Hilschmann and Craig, Proc. natn. Acad. Sci. U.S.A., 53, 1403; 1965). What is agreed is that separate genes code for $\mathrm{C}$ and $\mathrm{V}$ regions and that there is only a single $C$ gene for a given constant region (Ig class or subclass) sequence. The question may therefore be reframed in terms of $V$ genes: are all the conceivable $V$ genes carried in the genome or are they somatically generated?

Perhaps the strongest argument against the germ line theory is the existence of allelic markers and species -specific amino acid sequences in $\mathrm{V}$ regions. But this argument is inferential and it has long been the hope that direct experimental evidence could be brought to bear on the question. This now seems to be provided by Premkumar, Shoyab and Williamson (Proc. natn. Acad. Sci. U.S.A., 71, 99; 1974). In principle, the authors have isolated heavy chain messager RNA and have estimated by hybridisation the number of $C$ and $V$ genes in isolated DNA. Their results, they argue, firmly support the germ line theory.

An absolute prerequisite for this type of experiment is that the isolated messenger RNA is pure and labelled at high specific activity so that hybridisation can be done in great excess of DNA. In order to achieve this, cells from mouse myeloma MOPC 315 were labelled in vitro with radioactive uridine and cytidine, and then mRNA for the $x$ chain was isolated from the poly(A). containing fraction taking advantage of its specific interaction with immunoglobulin (Stevens and Williamson, Proc. natn. Acad. Sci. U.S.A., 70, 1127; 1973; J. molec. Biol., 78, 517; 1973). The resulting RNA sedimented largely as a single peak at $16-17 \mathrm{~S}$ and, after sonication to $6-7 \mathrm{~S}$, was hybridised with about a $10^{7}$ excess of cold DNA, also sonicated to $6.4 \mathrm{~S}$.

Using whole mouse embryo (the actual age is not given) as a source of DNA, a biphasic $C_{o} t$ curve resulted. Approximately $70 \%$ of the input RNA was hybridised at the maximum, and $20 \%$ was found in the low $C_{o} t$ transition. It is here that the authors make a crucial assumption, namely that the low $C_{o} t$ transition ( $C_{o} t \frac{1}{2}$ of 1.5) corresponds to hybridisation of $V$ genes and the high $C_{o} t$ transition $\left(C_{o} t \frac{1}{2}\right.$ of $\left.10^{3}\right)$ represents hybridisation to $C$ genes. If this assumption is wrong, then so are the conclusions. Their reasons for making this assumption are that the RNA is pure, and that the ratio of hybridisation found in the two regions of the $C_{o} t$ curve $(0.29)$ is close to the expected figure of 0.25 . The possible occurrence in the isolated RNA of RNA sequence stretches which do not code for $\mathrm{C}$ or $\mathrm{V}$ region sequences is obviously a source of uncertainty.

Similar, but not quite as impressive, results were obtained when BALB/C spleen or myeloma MOPC 315 DNA was used for hybridisation. Given the basic assumption made, the $C_{o} t \frac{1}{2}$ values correspond to 4-8 $C$ genes and about $5,000 \mathrm{~V}$ genes.

Although sequence analysis indicates considerable cross-homology of mouse $\mathrm{V}_{\mathrm{H}}$ regions, the figure of $5,000 V_{\mathrm{H}}$ genes if accepted, must be a lower estimate. If, as the authors assume, that there are an equal number of $V_{\mathrm{L}}$ genes and that $V_{L}$ and $V_{H}$ regions can associate at random, then there is sufficient information for at least $2.5 \times 10^{7}$ antibody-combining sites. Even allowing for inefficiency, this would seem to be a high enough figure to account for antibody diversity. The authors conclude that their data "obviates the necessity to search for somatic generators of diversity. Questions concerning the maintenance and control of a large $V$-gene pool in the germ line and the expression of $V$ genes by interaction with $C$ genes can now be brought more clearly into focus".

Until the fraction of RNA hybridising at the low $C_{o} t \frac{1}{2}$ transition is actually identified as specifying $\mathrm{V}$ region, however, proponents of the somatic theory are unlikely to be satisfied and the argument will continue with unabated force.

\section{Sense of supercoils in closed circular DNA}

\section{from a Correspondent}

AN interesting study on the sedimentation of denatured closed circular DNAs with different degrees of strand interwinding has been made by Schmir, Révet and Vinograd (J. molec. Biol., 82, 34-35; 1974). From their results these authors have deduced the absolute sense of the supercoils in closed circular DNA and are able to confirm that the binding of intercalating dyes unwinds. rather than winds up, the DNA helix.

Double stranded DNA in the form of closed circular superhelices was first discovered in extracts from polyoma virus in 1963, and has subsequently been shown to exist in a wide variety of organisms. The biological significance of the closure is not yet clear but these molecules have a number of interesting structural and chemical properties. The superhelical structure imposes upon the DNA molecule a topological restraint usually expressed by saying that, for a given molecule, the total number of rotations of one strand about the other, the topological winding number, is invariant. Thus a modification in the number of turns in the basic double helix, for example by changing the ionic strength of $p \mathrm{H}$ of the environment, or by the addition of intercalating dyes, is accompanied by an equal and opposite change in the number of superhelical turns. The introduction of a single scission into one of the DNA strands results in a circular DNA with no superhelical structure. The superhelical DNAs sediment at a higher rate than the nicked DNAs, the exact value of the sedimentation coefficient, $s$, being a complex function of the superhelix density, $\sigma$ (the number of superhelical turns per ten base pairs).

Vinograd, Lebowitz, Radloff, Watson and Laipis (Proc. natn. Acad. Sci. U.S.A., 53, 1104; 1965) studied the change in $s$ for a DNA in which the topological winding density, $A \simeq 1$, as the $p \mathrm{H}$ of the environment is progressively increased and the DNA becomes more denatured. At $p \mathrm{H} \simeq 12.5$, the DNA duplex is completely denatured, extensive positive supercoiling occurs and the sedimentation coefficient in these conditions is 2.1 times greater than that of a single stranded, circular molecule having an equivalent molecular weight. This greatly increased sedimentation coefficient can therefore be attributed directly to the winding of one strand about the other.

In the new article, Schmir et al. extend these results by studying the sedimentation coefficients, at high $p \mathrm{H}$ values, of DNAs with different topological winding densities. A number of closed circular DNAs with $A$ values 